\title{
Analytic solutions of the magnetic annihilation and reconnection problems. I. Planar flow profiles
}

\author{
P. G. Watson ${ }^{\text {a) }}$ and I. J. D. Craig \\ The University of Waikato, Hamilton, New Zealand
}

(Received 13 June 1996; accepted 15 October 1996)

\begin{abstract}
The phenomena of steady-state magnetic annihilation and reconnection in the vicinity of magnetic nulls are considered. It is shown that reconnective solutions can be derived by superposing the velocity and magnetic fields of simple magnetic annihilation models. These solutions contain most of the previous models for magnetic merging and reconnection, as well as introducing several new solutions. The various magnetic dissipation mechanisms are classified by examining the scaling of the Ohmic diffusion rate with plasma resistivity. Reconnection solutions generally allow more favorable "fast" dissipation scalings than annihilation models. In particular, reconnection models involving the advection of planar field components have the potential to satisfy the severe energy release requirements of the solar flare. The present paper is mainly concerned with magnetic fields embedded in strictly planar flows - a discussion of the more complicated three-dimensional flow patterns is presented in Part II [Phys. Plasmas 4, 110 (1997)]. (C) 1997 American Institute of Physics. [S1070-664X(97)03001-2]
\end{abstract}

\section{INTRODUCTION}

Magnetic reconnection is thought to be responsible for the explosive energy release observed in solar flares, tokamak disruptions and magnetic substorms in the geomagnetic tail. In such phenomena complicated magnetic field structures undergo a global simplification as fieldlines are cut and rejoined at magnetic null points. The released magnetic energy is converted into either the kinetic energy of ejected plasma or the thermal energy of resistively heated gas. An important theoretical problem is reconciling the explosive collapse of the field with the extremely low resistivities of typical plasmas.

As the magnetohydrodynamic (MHD) equations are highly nonlinear, the chances of finding an analytic description of reconnection seem remote. Accordingly, semianalytic or numerical approaches have traditionally been the preferred methods of obtaining solutions. Heuristic semianalytic solutions can certainly provide important insights into the problem, but their validity is always questionable. At first sight numerical simulations seem to provide the only self-consistent means of tackling the apparently intractable equations. Typical simulations ${ }^{1}$ however, are limited by unrealistic resistivities and the implementation of sensible boundary conditions-particularly in "open" planar geometries. $^{2}$

Motivated by these considerations, there has been a recent surge in the search for an analytic description of magnetic merging. Recent studies ${ }^{3-7}$ have generalized the well known magnetic annihilation solutions based on the ansatz of stagnation point flow. ${ }^{8,9}$ More significantly it has been shown that exact families of reconnection solutions can be constructed in both two and three dimensions. ${ }^{10-12}$ These display the essential characteristics of "fast" energy release-specifically, the collapse of the field to small length scales as required by an Ohmic heating rate that scales inde-

a)Electronic mail: pgwatson@hoiho.math.waikato.ac.nz pendently, or as some negative power, of the plasma resistivity. Existing two-dimensional (2-D) and three-dimensional (3-D) models suggest that extremely large external pressures are required to contain magnetically intense, flux pile-up regions close to the neutral point. A key question is whether fast reconnection solutions can be found which alleviate this difficulty.

The aim of this paper, and its companion paper, Watson and Craig, ${ }^{13}$ hereafter referred to as Part II, is to develop magnetic annihilation/reconnection solutions within the framework of incompressible, steady-state MHD, and to assess their possibilities as fast energy dissipation mechanisms. Although we recognize that a catastrophic event like a solar flare will involve a breakdown of the fluid approximation, we would argue that the MHD collapse to small length scales provides a necessary precursor to rapid energy release. We begin by considering only the simplest inviscid magnetic annihilation solutions, ${ }^{3-9}$ but show, by means of a general superposition argument, that these can be developed into fully reconnective models. In particular, we recover the reconnection solution of Craig and Henton, ${ }^{10}$ hereafter referred to as $\mathrm{CH}$, as well as generating several new quasi-steady reconnection models.

In Sec. II we introduce the MHD equations and describe our approach to developing analytic solutions. Only planar flows are considered in this paper and there are three families of solutions to discuss, each one given by setting a different component of the flow to zero. These solutions are analyzed in detail in Secs. III, IV and V. Our conclusions are summarized in Sec. VI.

\section{THE BASIC EQUATIONS}

The equations that govern the behavior of a magnetized incompressible fluid can be written in the following nondimensional form: 


$$
\begin{aligned}
& \frac{\partial \mathbf{v}}{\partial t}+(\mathbf{v} \cdot \nabla) \mathbf{v}=-\nabla p+(\nabla \times \mathbf{B}) \times \mathbf{B}, \\
& \frac{\partial \mathbf{B}}{\partial t}=\nabla \times(\mathbf{v} \times \mathbf{B})+\eta \nabla^{2} \mathbf{B}, \\
& \nabla \cdot \mathbf{B}=0, \quad \nabla \cdot \mathbf{v}=0,
\end{aligned}
$$

where the equations have been non-dimensionalized with respect to typical coronal parameters by scaling lengths with a length-scale $L_{c}$, magnetic fields with the background field strength $B_{c}$, velocities with the Alfvén speed $v_{A}$ $=B_{c} /(8 \pi \rho)^{1 / 2}$, time with the Alfvén travel time $\tau_{A}$ $=L_{c} / v_{A}$ and gas pressure with $B_{c}^{2} / 8 \pi$. The dimensionless resistivity, $\eta$, is given by

$$
\eta=\frac{\bar{\eta}}{v_{A} L_{c}}=\frac{M_{A}}{R_{m}},
$$

where $\bar{\eta}$ is the physical resistivity, $R_{m}$ is the magnetic Reynolds number and $M_{A}=v / v_{A}$ is the Alfvén Mach number. It is clear that the MHD system is invariant to translations and rotations of the coordinate system.

The gas pressure can be removed from this system of equations by taking the curl of the equation of motion, to give

$$
\frac{\partial \boldsymbol{\omega}}{\partial t}+(\mathbf{v} \cdot \nabla) \boldsymbol{\omega}-(\boldsymbol{\omega} \cdot \nabla) \mathbf{v}=(\mathbf{B} \cdot \nabla) \mathbf{J}-(\mathbf{J} \cdot \nabla) \mathbf{B},
$$

where $\mathbf{J}=\nabla \times \mathbf{B}$ is the electric current and $\boldsymbol{\omega}=\nabla \times \mathbf{v}$ is the vorticity. Assuming a plausible solution has been constructed for the magnetic and velocity fields, the gas pressure distribution is determined from the primitive (uncurled) form of the momentum equation.

\section{A. The rate of resistive energy dissipation}

The MHD system described above is conservative apart from resistive energy losses. The global energy of the fluid is dissipated at the rate

$$
\mathscr{W}_{\eta}=\left\langle\eta J^{2}\right\rangle=\eta \int J^{2} d V
$$

and unless very large currents are set up this rate is physically negligible in typical coronal plasmas. This is a consequence of the smallness of the resistivity- $\eta$ is typically $O\left(10^{-12}\right)$ in collision dominated gases. The upshot is that the magnetic field must possess very small length scales if the currents are to be large enough to dissipate a significant amount of energy. Although plasma instabilities can raise the effective collision frequency, causing enhancements in the resistivity by factors exceeding $10^{4}$ (see Parker, ${ }^{14}$ p. 783), small length scales are still necessary for appreciable energy release.

The most severe constraints on the Ohmic dissipation rate are provided by the solar flare. Consider a coronal field of strength $B_{c}=100 \mathrm{G}$ occupying the volume $V=L_{c}^{3}$ where $L_{c}=10^{9.5} \mathrm{~cm}$. Then a modest reduction in the field of a few Gauss is sufficient to produce a typical flare energy of say $10^{29}$ to $10^{30}$ ergs. This energy is liberated within a few hundred seconds and so the power output must average $10^{27}$ to $10^{28}$ ergs per second.
To understand the severity of this constraint we first note that $\mathscr{W}_{\eta}$ is conveniently calibrated using the coronal magnetic energy $\left\langle B_{c}^{2} / 8 \pi\right\rangle$ divided by the coronal Alfvén time $\tau_{A}=L_{c} / v_{A}$. For the typical speed $v_{A} \simeq 10^{8.5} \mathrm{~cm} / \mathrm{s}, \tau_{A}=10 \mathrm{~s}$, and so $\mathscr{W}_{\eta}$ is measured in units of $10^{30}$ ergs per second. It follows that $\mathscr{W}_{\eta}$ for a typical coronal resistivity $\eta=\eta_{c}$ must be of order $10^{-3}$ to achieve an output power comparable to a flare. In particular, for $\eta_{c}=10^{-12}$, we obtain the severe requirement that the dimensionless current must build up to the level $\left\langle J^{2}\right\rangle \sim 10^{9}$.

\section{B. Magnetic annihilation solutions}

We begin by presenting steady-state magnetic annihilation solutions in which anti-parallel fields are swept together by the flow. Reconnection solutions are then constructed from annihilation models by invoking the method of Sec. II C. Although the annihilation of the magnetic field embedded in planar flows has been well studied by a number of previous authors, ${ }^{3-9}$ we feel it is important to re-cap the various solutions here, as they provide the basis for our reconnection solutions-we also emphasize some difficulties associated with these solutions.

Specializing to the case of one component fields, with the field directed in the $z$-direction, the condition $\nabla \cdot \mathbf{B}=0$ implies that the field must be of the form $\mathbf{B}=Z(x, y) \hat{\mathbf{z}}$. We make the further simplification that $\mathbf{B}$ is independent of $y$, so that

$$
\mathbf{B}=[0,0, Z(x)] .
$$

Note that for this type of field $(\mathbf{B} \cdot \nabla) \mathbf{J}-(\mathbf{J} \cdot \nabla) \mathbf{B}=0$.

What types of flow can maintain this magnetic field against resistive diffusion? To retain as much generality as possible we initially make no assumptions about the form of the flow and take

$$
\mathbf{v}=[U(x, y, z), V(x, y, z), W(x, y, z)] .
$$

The three equations we must satisfy are the steady-state momentum equation,

$$
(\mathbf{v} \cdot \nabla) \boldsymbol{\omega}-(\boldsymbol{\omega} \cdot \nabla) \mathbf{v}=(\mathbf{B} \cdot \nabla) \mathbf{J}-(\mathbf{J} \cdot \nabla) \mathbf{B},
$$

the induction equation

$$
(\mathbf{v} \cdot \nabla) \mathbf{B}-(\mathbf{B} \cdot \nabla) \mathbf{v}=\eta \nabla^{2} \mathbf{B},
$$

and the continuity equation,

$$
\nabla \cdot \mathbf{v}=0 \text {. }
$$

On substituting for $\mathbf{v}$ and $\mathbf{B}$ into Eqs. (1)-(3) one finds that $\mathbf{v}$ must be of the form

$$
\mathbf{v}=[-a(x), b(x) y+f(x), c(x) z+g(x, y)],
$$

where $a^{\prime}(x)-b(x)-c(x)=0$, while the function $Z(x)$ must satisfy

$$
\eta Z^{\prime \prime}+a(x) Z^{\prime}+c(x) Z=0 .
$$

Allowable forms for the functions $a, b, c, f, g$ must be determined from the momentum equation. Note, in agreement with Phan and Sonnerup, ${ }^{4}$ that it is not necessary to make the ansatz of stagnation point flow. ${ }^{3,5-9}$ 


\section{Constructing reconnection solutions}

In what follows we exploit the fact that fields defined by magnetic annihilation models can be used as prototypes in the construction of magnetic reconnection solutions. Noting the remarkable symmetry between $\mathbf{v}$ and $\mathbf{B}$ in Eqs. (1) and (2) - a symmetry broken only by the resistive diffusion term-we assume reconnection solutions of the form

$$
\begin{aligned}
& \mathbf{v}=\mathbf{v}_{a}+\lambda \mathbf{B}_{a}, \\
& \mathbf{B}=\lambda \mathbf{v}_{a}+\mathbf{B}_{a},
\end{aligned}
$$

where $\mathbf{v}_{a}$ and $\mathbf{B}_{a}$ are the velocity and magnetic fields of the annihilation solution and $\lambda$ is a constant.

Substituting these forms into Eqs. (1)-(3) we find that the momentum and continuity equations are automatically satisfied, while the induction equation yields

$$
\left(1-\lambda^{2}\right)\left\{\left(\mathbf{v}_{a} \cdot \nabla\right) \mathbf{B}_{a}-\left(\mathbf{B}_{a} \cdot \nabla\right) \mathbf{v}_{\mathbf{a}}\right\}=\eta \nabla^{2} \mathbf{B}_{a}+\lambda \eta \nabla^{2} \mathbf{v}_{a} .
$$

This equation can be satisfied by an annihilation solution with a new effective velocity given by $\left(1-\lambda^{2}\right) \mathbf{v}_{a}$, provided $\nabla^{2} \mathbf{v}_{a}=0$. As we will see, solutions that meet this constraint do exist, and because of the added complexity of their flow/ field topology they allow for the possibility of magnetic reconnection.

Suppose however, that $\nabla^{2} \mathbf{v}_{a}$ does not vanish identically. If the annihilation velocity field $\mathbf{v}_{a}$ contains only global length scales then the offending term makes only a negligible $O(\eta)$ contribution to the induction equation. Neglecting this term yields a model which, although not formally exact, can be regarded as a quasi-steady reconnection solution for all practical purposes since any evolution of the quasi-steady solution only occurs very slowly, on the time scale $t \sim \eta^{-1}$.

Finally, we mention another interpretation of the reconnection solutions: they can be thought of as describing the non-linear disturbance of some quiescent equilibrium - an interpretation central to $\mathrm{CH}$. Specifically, we regard the field components $\mathbf{v}_{d}=\lambda Z(x) \hat{\mathbf{z}}$ and $\mathbf{B}_{d}=Z(x) \hat{\mathbf{z}}$ as being superposed onto the background quiescent solution $\mathbf{v}_{q}=\mathbf{v}_{a}$, $\mathbf{B}_{q}=\lambda \mathbf{v}_{a}$. All flows in the background field are constrained to the fieldlines but departures from potential fields ${ }^{11}$ are possible, at least for quasi-steady solutions. A key feature of the reconnection analysis is that the superposed "displacement field" can be normalized relative to the background field in any convenient manner. This freedom is exploited in Sec. III D.

\section{The family of models}

Our basic aim is to demonstrate that simple, global velocity fields can naturally support localized resistive dissipation in the fluid. Since we restrict our attention to purely planar flows it is natural to classify solutions in terms of whether planar or non-planar field components are advected by the flow. In fact we shall see that it is only planar components which are magnified by advection towards the neutral line-and which can lead to fast reconnection solutions. We begin therefore by reviewing annihilation solutions in which fieldlines, lying in the plane of the flow, are swept together into the current layer.

\section{DISSIPATION OF PLANAR FIELD COMPONENTS:} FLOWS WITH $V=0$

\section{A. Annihilation solutions}

When the $y$-component of the flow vanishes one must take

$$
\mathbf{v}=\left[-a(x), 0, a^{\prime}(x) z+g(x, y)\right],
$$

in order to support an annihilation solution with $\mathbf{B}=Z(x) \hat{\mathbf{z}}$. Equation (5) shows that $g(x, y)$ has no influence on the magnetic field-it merely represents distortions of the basic background flow $^{3-9}$ dictated by $a(x)$-and so we set $g(x, y)=0$ in what follows. Substituting the expression for $\mathbf{v}$ into the momentum equation implies that

$$
a^{\prime} a^{\prime \prime}=a a^{\prime \prime \prime} \text {. }
$$

This equation has solutions of the form

$$
a(x)=\left\{\begin{array}{l}
\alpha_{1} x+\alpha_{0}, \\
\alpha_{1} \sin (k x)+\alpha_{0} \cos (k x), \\
\alpha_{1} \sinh (k x)+\alpha_{0} \cosh (k x),
\end{array}\right.
$$

where $k$ is a constant, so that there are three allowable types of flow. The parameter $\alpha_{0}$ can be set to zero by suitably relabelling the axes.

The magnetic field component $Z(x)$ is determined by the equation

$$
\eta Z^{\prime}+a(x) Z=E_{0},
$$

where $E_{0}$ is a constant that can be identified with the $y$-component of the background electric field. Solutions for $Z(x)$ can always be expressed in quadrature form by defining

$$
H^{ \pm}(x)=\exp \left[ \pm \frac{1}{\eta} \int^{x} a(u) d u\right]
$$

and writing

$$
Z(x)=\frac{E_{0}}{\eta} H^{-}(x) \int^{x} H^{+}(u) d u .
$$

This form describes the flux pile-up annihilation solutions discussed at length in the literature. ${ }^{3-9}$

In the case of the linear flow profile the solution can be expressed in terms of known functions (see $\mathrm{CH}$ ). We introduce the Dawson function ${ }^{15}$

$$
\operatorname{daw}(x)=\int_{0}^{x} \exp \left(t^{2}-x^{2}\right) d t,
$$

which increases as $x-2 x^{3} / 3$ for small $x$, peaks when $\operatorname{daw}(x) \simeq 0.541$ at $x \simeq 0.924$, before declining monotonically, as $1 /(2 x)$, for large $x$.

The general form of the linear flow is

$$
\mathbf{v}=[-\alpha x, 0, \alpha z],
$$

and the solution of (11) in this case is given by

$$
Z(x)=\frac{E_{0}}{\eta \mu} \operatorname{daw}(\mu x)+C_{1} \exp \left(-\mu^{2} x^{2}\right),
$$

where $\mu^{2}=\alpha /(2 \eta)$. This linear velocity pattern represents a stagnation point flow in the $x-z$ plane. 


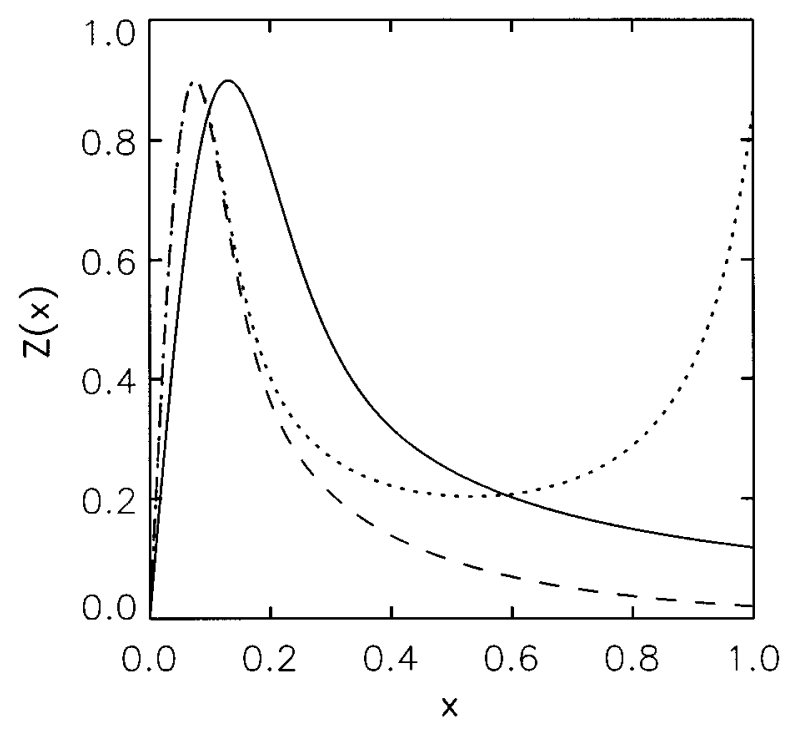

FIG. 1. Plots of magnetic field $Z(x)$ versus $x$ for the case of the annihilation of planar field components. Solutions are for the parameter set $\alpha=1, k$ $=3, \eta=0.01$ and the three different velocity profiles: linear $(-)$, trigonometric $(\cdots)$ and hyperbolic $(---)$. These antisymmetric solutions have been scaled to have the same peak magnetic field in the sheet near the null. The magnetic fields for both the linear and hyperbolic flows decay monotonically near the outer boundary, while the solution for the trigonometric flow starts to increase again for this value of $k$.

Solutions for the sinusoidal and hyperbolic flow patterns must be determined numerically. Although the trigonometric solution can be fundamentally different ${ }^{7}$ from the other two flows if $k>\pi$, as it then has multiple stagnation points, we restrict our attention to the case $k<\pi$ for simplicity.

Figure 1 compares the magnetic fields for the three different types of inflow profile. Although the behavior of the magnetic field in the outer region may differ, it is clear that the field in the vicinity of the origin is similar for all three flows. In each case the inner field is confined to strong sheets on either side of the magnetic null, as expected for flux pile-up solutions. Note that these solutions have been scaled to have the same peak field in the sheet for the purposes of comparison. To determine how each solution scales with resistivity we must fix the value of the field at the boundary while allowing $\eta$ to vary.

\section{B. Are fast annihilation solutions possible?}

To investigate whether fast dissipation is possible we first consider the Dawson function solution under the traditional flow symmetries, that is $C_{1}=0$. The behavior of the Dawson function for large arguments implies that $Z(1) \simeq E_{0} / \alpha$ as $\eta \rightarrow 0$. This means that the constant $E_{0}$, which provides a measure of the flux annihilation rate, can be chosen independently of $\eta$ by fixing the magnetic field strength at the boundaries. The maximum field strength in the sheet $Z_{s}$, which occurs at $x_{s} \simeq 0.924 \sqrt{2 \eta / \alpha}$ scales as $\eta^{-1 / 2}$, while the current density $J_{s}$, which is proportional to

$$
Z^{\prime}=\frac{E_{0}}{\eta}\{1-2 \mu x \operatorname{daw}(\mu x)\},
$$

scales as $\eta^{-1}$. These scalings imply that the Ohmic heating rate,

$$
\mathscr{W}_{\eta}=\eta\left\langle J^{2}\right\rangle \simeq \eta J_{s}^{2} d V_{s} \simeq O\left(\eta^{-1 / 2}\right),
$$

is fast for this type of annihilation solution. The same behavior for solutions with trigonometric and hyperbolic flow profiles can be deduced from numerical computations. Thus we conclude that this family of models achieves a fast energy release by allowing the magnetic field to "pile-up" into thin sheets on either side of the magnetic null point.

There is however, a major difficulty with such flux pile-up solutions. The gas pressure is given by

$$
p=p_{0}-\frac{1}{2}\left(a^{2}+a^{\prime 2} z^{2}+Z^{2}\right)+\frac{1}{2} a a^{\prime \prime} z^{2} .
$$

It follows that since $\left|Z_{s}\right|$ scales as $\eta^{-1 / 2}$, the background pressure, $p_{0}$, must scale as $\eta^{-1}$ to avoid non-physical negative pressures. Thus the super-fast dissipation rate is achieved at the cost of building up unphysically large pressures within the annihilation region. Obviously, it makes little physical sense for $p_{0}$ to exceed the external hydromagnetic pressures that power the merging process-say, the magnetoconvection pressures associated with photospheric sunspot motions. And since $p_{0}$ essentially determines the pressure on the boundary it appears difficult to reconcile any of these solutions with a low-beta coronal plasma in the far field.

These objections can be countered to some extent by postulating that the solution is sandwiched between magnetically dominant external boundary regions which lie outside the reconnection region. Some support for this notion is provided by the sinusoidal velocity solution, which shows that we are free to choose a wave number $k \lesssim \pi$ that allows $Z$ to build up on the outer boundary. Such a solution is shown by the dotted curve in Fig. 1. We see that low pressure coronal conditions can now be approximated in the far field at the cost of introducing a strong dissipation region at the outer boundary. In fact the outer boundary current, as indicated by the steep gradient in $Z$, is directly comparable in strength to the central current sheet. Of course, the extreme pressure variations within the reconnection region remain.

We conclude that flux pile-up annihilation solutions, although formally fast, generally run up against severe physical difficulties. An encouraging feature of the reconnection solutions described below is that the pressure problem can be overcome far more naturally without compromising the fast dissipation rate.

\section{Reconnection solutions}

In the case of the linear velocity profile the superposition method of Sec. II C yields the exact solution

$$
\begin{aligned}
& \mathbf{v}=\left[\begin{array}{lll}
-\alpha x, & 0, & \alpha z+\lambda Z(x)
\end{array}\right], \\
& \mathbf{B}=\left[\begin{array}{lll}
-\lambda \alpha x, & 0, \lambda \alpha z+Z(x)], \\
Z(x)=\frac{E_{0}}{\eta \bar{\mu}} \operatorname{daw}(\bar{\mu} x)+C_{1} \exp \left(-\bar{\mu}^{2} x^{2}\right),
\end{array}\right.
\end{aligned}
$$



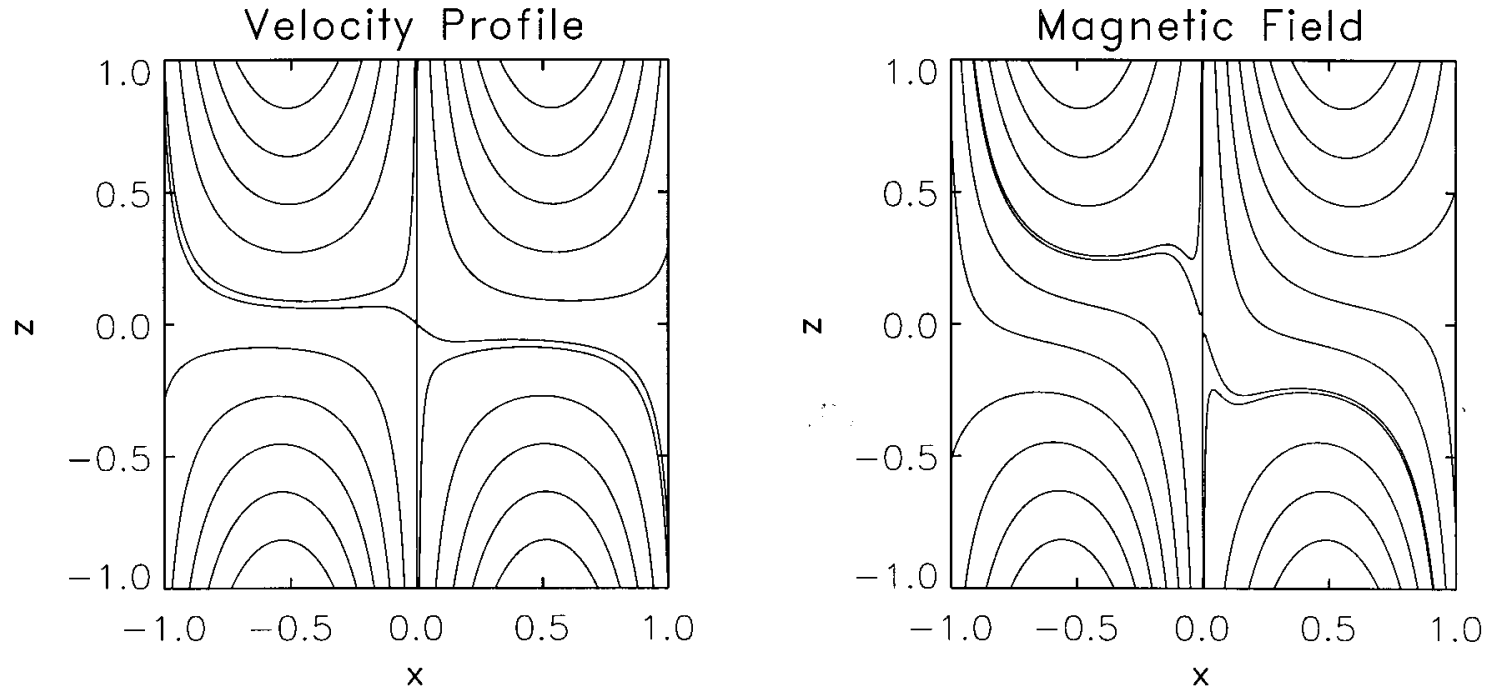

FIG. 2. Contour plots of streamlines and magnetic fieldlines for the superposed trigonometric flow solution with $\alpha=1, k=3, \eta=0.01, E_{0}=0.1$ and $\lambda=0.5$. This solution has a strong current sheet contiguous with the plane $x=0$, which is a separatrix for both the flow and the field. The other separatrices for the velocity and magnetic fields do not coincide. Notice in particular that there is flow across the curved magnetic field separatrix, a clear signature that magnetic reconnection is occurring.

where $\bar{\mu}=\sqrt{\left(1-\lambda^{2}\right) \alpha /(2 \eta)}$. An alternate derivation and detailed analysis of solution (18) is given by $\mathrm{CH}$. A new feature of the present analysis however, is that highly accurate quasi-steady reconnection solutions can also be deduced for the sinusoidal and hyperbolic flow profiles, provided $k$ $\ll O\left(\eta^{-1 / 2}\right)$.

A superposed solution with a sinusoidal inflow profile is shown in Fig. 2. The fact that there is flow across the curving separatrix of the field - the separatrix not contiguous with the current layer-confirms the solution as reconnective. This is also reflected by the presence of strong shearing motions across the current layer. These features are not restricted to sinusoidal flow solutions: they are generic to all three flow profiles.

\section{Solution scalings with plasma resistivity}

Although the reconnection solutions scale in exactly the same way as the annihilation models, they can be given a completely different physical interpretation, as discussed in Sec. II C. Specifically, to avoid unbounded Ohmic dissipation losses in the limit of small $\eta$, we identify $\mathbf{v}_{d}=\lambda Z(x) \hat{\mathbf{z}}$ and $\mathbf{B}_{d}=Z(x) \hat{\mathbf{z}}$ as disturbance fields superposed on the quiescent solution $\mathbf{v}_{q}=\mathbf{v}_{a}, \mathbf{B}_{q}=\lambda \mathbf{v}_{a}$. Since the plasma pressure is now given by

$$
p=p_{0}-\frac{1}{2}\left(a^{2}+a^{\prime 2} z^{2}+Z^{2}\right)+\frac{1-\lambda^{2}}{2} a a^{\prime \prime} z^{2}-\lambda a^{\prime} z Z,
$$

we assume that the pressure of the disturbance field $Z(x)$ cannot overwhelm the background field contribution $\mathbf{B}_{q}=\lambda \mathbf{v}_{a}$. In practice, this is tantamount to bounding the field $Z_{s}$ in the sheet at a level determined by the distant field. That $Z_{s}$ must eventually saturate also follows from the re- quirement that the displacement velocity field magnitudewhich is absent in the pure annihilation solution-must be bounded by the speed of light $c$.

The basic idea is illustrated in Fig. 3. The amplitude of the disturbance field on the outer boundary is chosen small

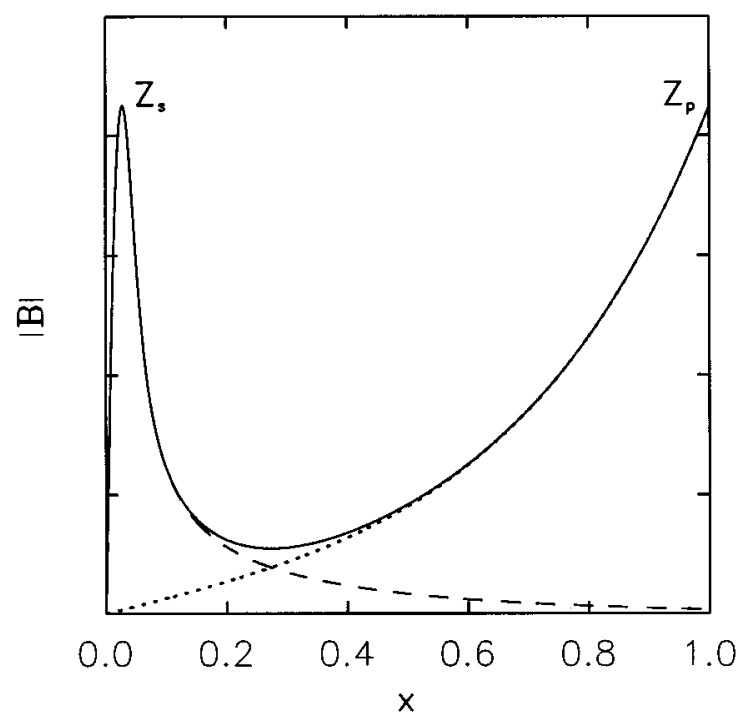

FIG. 3. A plot of the magnitude of the magnetic field along the $x$-axis for a superposed solution with a hyperbolic velocity inflow profile. The solid line gives the total magnitude of the field, while the dashed and dotted lines represent the contributions from the disturbance field, $Z$, and the quiescent background field, $\lambda \mathbf{v}_{a}$, respectively. The amplitude of the disturbance field is completely arbitrary, but we argue from physical considerations that the pressure forces generated in the sheet cannot greatly exceed the forces on the boundary that drive the reconnection process. This suggests that the field in the sheet, $Z_{s}$, must be limited by the field $Z_{p}$ on the boundary. 
enough so that the flux pile-up layer is no longer more intense magnetically than the "far field" on the boundaries $x \simeq \pm 1$ that sandwich the reconnection region. In other words, in recognition of the fact that magnetoconvective footpoint forces ultimately drive the reconnection, we take $\left|Z_{s}\right|$ to be bounded by the magnitude $\left|Z_{p}\right|$ of the photospheric field at the boundary. The Ohmic power output, namely

$$
P_{o}=\eta_{c}\left\langle J^{2}\right\rangle \simeq \eta_{c} J_{s}^{2} d V_{s} \simeq \eta_{c}{ }^{1 / 2} Z_{s}{ }^{2},
$$

where $\eta_{c}$ is the coronal resistivity, then builds up only until the sheet field strength $Z_{s}$ approaches $Z_{p}$. Since $Z_{s}$ cannot exceed $Z_{p}$ the maximum dissipation rate saturates at the limit $P_{o} \simeq \eta_{c}{ }^{1 / 2} Z_{p}^{2}$ The pressure is now bounded by the limit $p \sim Z_{p}^{2} / 2$ and so the unphysical gas pressure distribution of the annihilation model is avoided.

It is important to ask whether the pressure problem has been overcome at the drastic cost of making the solution "slow." In fact, the solution remains fast provided the level of the disturbances are small enough to maintain the limit $Z_{s} \sim Z_{p}$. The question is really whether the limiting Ohmic dissipation rate $P_{o}=\eta_{c}{ }^{1 / 2} Z_{p}{ }^{2}$ is sufficient to power a flare. Taking $Z_{p}=10^{1.5}$-which corresponds to a photospheric field of order $3000 \mathrm{G}$-yields $P_{o}=O\left(10^{-3}\right)$ which, as discussed in Sec. II B, is quite sufficient to power a sizable event. The extreme conditions of the flare are now reflected in the large displacement velocities-at $30 v_{A}$ these are now approaching an appreciable fraction of the speed of light $c$. These conditions are significantly eased however, if $\eta_{c}$ is enhanced by micro-instabilities in the sheet. A modest increase, say by a factor of $10^{4}$, implies that $Z_{s}$ of order unity is quite sufficient for a moderate flare. Thus the model appears feasible even assuming fairly conservative saturation limits.

Finally we mention an apparent difficulty that arises with the reconnection (and annihilation) solutions if we consider solutions that deviate from perfect anti-symmetry. In this case it appears that the magnetic field should scale according to $\left|Z_{s}\right| \simeq C_{1} \sim \exp (1 / \eta)$. We will show in Part II however, that this unphysical behavior is an artifact of imposing strict two-dimensionality on the flow.

\section{DISSIPATION OF NORMAL FIELD COMPONENTS: FLOWS WITH $W=0$}

\section{A. Annihilation solutions}

We now consider a second class of annihilation solutions formed by setting $W \equiv 0$ in the expression for the velocity profile, Eq. (4). The flow now has the form

$$
\mathbf{v}=\left[-a(x), a^{\prime}(x) y+f(x), 0\right],
$$

where $f(x)$ can once again be set to zero as it only represents a distortion of the basic flow pattern that does not affect the magnetic field. To satisfy the momentum equation the function $a(x)$ must obey the relation $a^{\prime} a^{\prime \prime}=a a^{\prime \prime \prime}$. This equation is the same as that covered by the discussion of Sec. III A, and one obtains linear, sinusoidal and hyperbolic profiles for the inflow $a(x)$. Turning to the induction equation, we find that the magnetic field component $Z(x)$ is determined by

$$
\eta Z^{\prime \prime}+a(x) Z^{\prime}=0 \text {. }
$$

Solutions for the three possible choices of $a(x)$ are shown in Fig. 4.

If we assume a linear flow profile the magnetic field is maintained by a flow of the generic form

$$
\mathbf{v}=[-\alpha x, \alpha y, 0],
$$

where $\alpha$ is a constant. This velocity profile now represents a stagnation point flow in the $x-y$ plane. The solution of Eq. (22) may now be written as

$$
Z(x)=C_{1} \operatorname{erf}(\mu x)+C_{2},
$$

see Besser et al. ${ }^{6}{ }^{2}$ where the constant $\mu$ is given by $\mu^{2}=\alpha /(2 \eta)$ and $C_{1}$ and $C_{2}$ are determined by the magnetic field on the boundaries $x= \pm 1$. Traditional annihilation solutions model the merging of perfectly anti-parallel fields, which would imply $Z(0)=0$, but this symmetry can be broken if the constant $C_{2}$ is non-zero.

\section{B. Superposed solutions}

A second class of solutions can be constructed by performing the superposition given in (6). For the linear flow we find that

$$
\begin{aligned}
& \mathbf{v}=[-\alpha x, \alpha y, \lambda Z(x)], \\
& \mathbf{B}=[-\lambda \alpha x, \lambda \alpha y, Z(x)], \\
& Z(x)=C_{1} \operatorname{erf}(\bar{\mu} x)+C_{2},
\end{aligned}
$$

where $\bar{\mu}=\sqrt{\left(1-\lambda^{2}\right) \alpha /(2 \eta)}$ provides an exact solution to the MHD equations as the condition $\nabla^{2} \mathbf{v}_{a}=0$ is satisfied. As before, the trigonometric and hyperbolic profiles lead to new solutions in the sense of Sec. II C. The key point is that - unlike the superposed solutions of Sec. III B-none of these solutions allow flow across the separatrices of the magnetic field, and hence they are not reconnective. Specifically, since $z$ is an ignorable coordinate in the present solutions, only planar reconnection can occur-and this can be overruled by noting that the streamlines and fieldlines coincide when they are projected onto planes of constant $z$.

\section{Solution scalings with plasma resistivity}

We examine the superposed solution with the linear flow profile first, as its scalings can be determined analytically. Equation (25) shows that the magnetic field is uniform outside the diffusion region. Therefore $J_{s}$ scales as $\eta^{-1 / 2}$, which implies the slow heating rate,

$$
\mathscr{H}_{\eta}=\eta\left\langle J^{2}\right\rangle \simeq \eta J_{s}^{2} d V_{s} \simeq O\left(\eta^{1 / 2}\right) .
$$

This behavior in fact accords with the traditional slow Sweet-Parker scaling. But whereas the approximate SweetParker model describes the annihilation of planar field, this solution provides an exact solution for the dissipation of normal field components. Numerical simulations reveal that the Ohmic heating rates for the trigonometric and hyperbolic flow solutions (both annihilation and superposed) obey exactly the same scaling law, so they too are slow dissipation solutions.

The absence of flux pile-up in these solutions-i.e. $|Z|_{\max }=O(1)$ - shows that there is no difficulty with the pressure profile, 


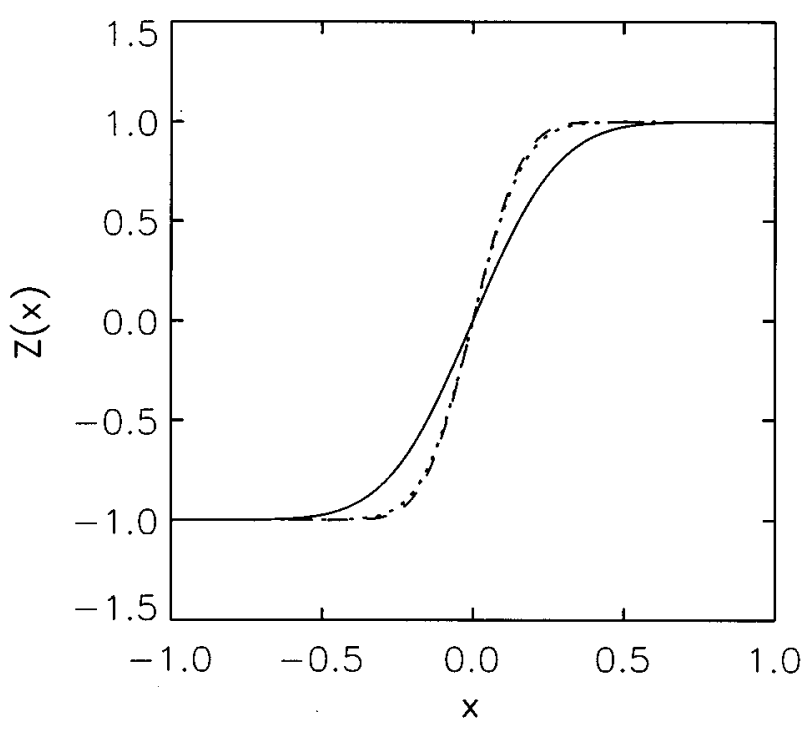

FIG. 4. Plots of the magnetic field $Z(x)$ versus $x$ for the annihilation of the normal field component. Plots for the three different velocity profiles, linear (一), trigonometric $(\cdots)$ and hyperbolic $(---)$ are shown. These antisymmetric solutions were generated with the parameter set $\alpha=1, k=3$, $\eta=0.05$ and the boundary conditions $Z(0)=0$ and $Z(1)=1$. Clearly the structure of the magnetic field is relatively insensitive to the form of the inflow profile.

$$
p=p_{0}-\frac{1}{2}\left(a^{2}+a^{\prime 2} y^{2}+Z^{2}\right)+\frac{1-\lambda^{2}}{2} a a^{\prime \prime} y^{2},
$$

where $a(x)$ corresponds to any one of the allowable flow profiles given in (10). It is clear that normal field components-unlike planar components-are not amplified by advection into the current layer.

\section{DISSIPATION OF TRANSVERSELY VARYING FIELDS: FLOWS WITH $U=0$}

\section{A. Annihilation solutions}

A final form of planar flow annihilation solution can be derived by setting $U=0$ in the expression for the fluid velocity. This implies that the velocity must be of the form

$$
\mathbf{v}=[0, b(x) y+f(x),-b(x) z+g(x, y)],
$$

in order to support an annihilation solution. This type of planar flow annihilation solution has not appeared in the literature before, so we shall retain $f$ and $g$ in order to determine their influence on the new flow profile. Substituting this expression for the velocity into the momentum equation we find that

$$
b(x)=\beta_{0}, \quad f(x)=\gamma_{0}, \quad g(x, y)=h(x)\left(y+\frac{\gamma_{0}}{\beta_{0}}\right)+\delta_{0},
$$

where $\beta_{0}, \gamma_{0}$ and $\delta_{0}$ are constants and $h(x)$ is an arbitrary function of $x$. The constants $\gamma_{0}$ and $\delta_{0}$ can always be set to zero by a suitable relabelling of the $y$ and $z$ coordinates, hence the general form of the flow pattern is given by

$$
\mathbf{v}=[0, \beta y,-\beta z+h(x) y]
$$

where we have dropped the subscript on $\beta$. This type of flow gives rise to a magnetic field component $Z(x)$ that must satisfy

$$
\eta Z^{\prime \prime}-\beta Z=0
$$

Equation (31) implies that there are three allowable field configurations,

$$
Z(x)=\left\{\begin{array}{l}
C_{1} x+C_{2}, \quad \beta=0, \\
C_{1} \sin (\mu x)+C_{2} \cos (\mu x), \quad \beta<0, \\
C_{1} \sinh (\mu x)+C_{2} \cosh (\mu x), \quad \beta>0,
\end{array}\right.
$$

where $\mu=\sqrt{|\beta| / \eta}$. Since only the trigonometric solution possesses field and current structures that are localized in the interior of the domain as $\eta \rightarrow 0$, we restrict our attention to this case.

The structure of this solution is somewhat unusual. The flow, which is confined to the $y-z$ plane, supports a magnetic field $\mathbf{B}=Z(x) \hat{\mathbf{z}}$ that varies in the $x$ direction. In any given plane of $x$ the magnetic field is uniform, while the flow resembles a 2D stagnation point flow. One separatrix of the flow is aligned with the direction of the magnetic field, while the orientation of the other is determined by the value of $h(x)$-the separatrices are orthogonal when $h(x)=0$.

The role of the $x$-axis has also changed for this solution. In the previous two sections the $x$-axis represented the inflow direction as well as the direction in which the field varied. Although it is still true that the field varies in this direction, the inflow direction is now along the $y$-axis (remember $\beta$ is negative). This implies that there are fine scale field variations on the inflow boundaries $y= \pm 1$.

\section{B. Superposed solutions}

Another exact solution can be developed by means of the usual superposition procedure, which yields

$$
\begin{aligned}
& \mathbf{v}=[0,-\beta y, \beta z+h(x) y+\lambda Z(x)], \\
& \mathbf{B}=[0,-\lambda \beta y, \lambda \beta z+\lambda h(x) y+Z(x)], \\
& Z(x)=C_{1} \sin (\bar{\mu} x)+C_{2} \cos (\bar{\mu} x),
\end{aligned}
$$

where $\bar{\mu}=\sqrt{\left|\left(1-\lambda^{2}\right) \beta\right| / \eta}$. The function $h(x)$ must now be a linear function of $x$ for the superposition constraint to be satisfied exactly.

In order to visualize the basic structure of this solution it is instructive to set $h(x) \equiv 0$ and let $Z(x)=\sin (\mu x)$, see Fig. 5. The streamlines and fieldlines are then confined to planes of constant $x$ and both have an X-type structure. When $\mu x$ $=n \pi$ the function $Z(x)$ vanishes and the streamlines and fieldlines coincide exactly, but for other values of $x$ one pair of the separatrices of the field and the flow become separated. There are three distinct separatrix planes in the problem: the plane $y=0$, which is a separatrix for both the flow and the field; and the two corrugated planes $z=\lambda \sin (\mu x) / \beta$ and $z=\sin (\mu x) /(\lambda \beta)$, which denote the other separatrix planes of the flow and field, respectively. This is a reconnective solution, as there is flow across the corrugated separatrix of the magnetic field. 


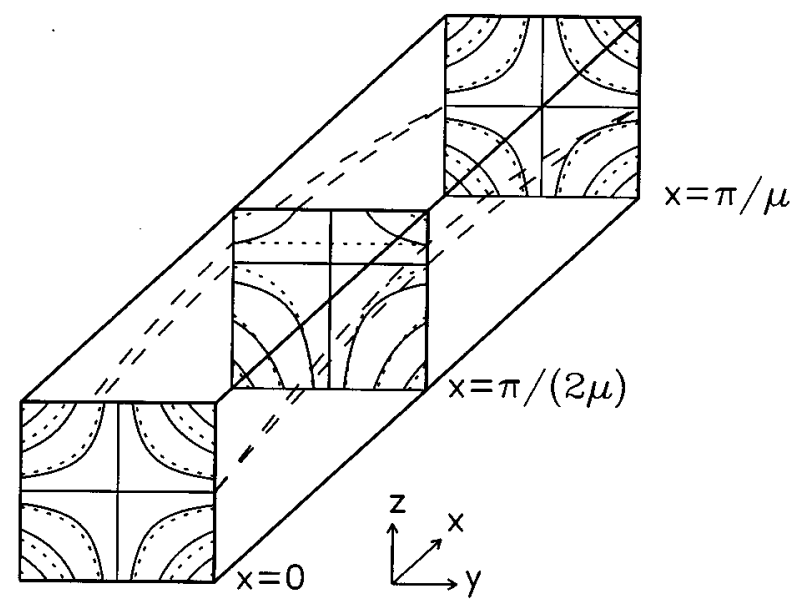

FIG. 5. A schematic representation of the velocity and magnetic fields for the superposed transversely varying field solution with $\mathbf{v}$ $=[0, \beta y,-\beta z+\lambda \sin (\mu x)]$ and $\mathbf{B}=[0, \lambda \beta y,-\lambda \beta z+\sin (\mu x)]$. Flow streamlines and magnetic fieldlines are confined to planes of constant $x$. Contours of the streamlines (solid contours) and fieldlines (dotted contours) are given for the three planes, $x=0, x=\pi /(2 \mu)$ and $x=\pi / \mu$. In the planes $x=0, \pi / \mu$ the flow and the field coincide. The plane $x=\pi /(2 \mu)$ is the plane where the velocity profile and the magnetic field are most out of phase. The dashed lines $z=\lambda \sin (\mu x) / \beta$ and $z=\sin (\mu x) /(\lambda \beta)$ represent the corrugated separatrix planes of the flow and the field, respectively.

\section{Solution scalings}

How do these solutions behave as $\eta$ is systematically reduced? Clearly the magnetic field for solution (33) oscillates more rapidly as $\eta \rightarrow 0$, but its maximum amplitude is unaffected. The field loses energy to Ohmic heating in the current sheets that separate alternate regions of the antiparallel field. Although the current in these sheets increases with decreasing $\eta$ the sheets also become narrower, so that they remain relatively weak heating sources. Despite the fact that the individual current sheets are weak, a fast global Ohmic heating rate, $\mathscr{W}_{\eta}$, is sustained due to the fact that the number of sheets increases as the resistivity is decreased, i.e.

$$
\mathscr{\mathscr { V }}_{\eta}=\eta\left\langle J^{2}\right\rangle=\eta \int_{-1}^{1} Z^{\prime 2} d x=O(1), \quad \text { as } \eta \rightarrow 0 .
$$

The magnetic field is maintained against these losses by virtue of the fact that it is embedded in a stretching flow.

We note that as well as being a fast dissipation solution this solution also appears to quite naturally overcome any gas pressure problem, as the pressure, given by

$$
\begin{aligned}
p= & p_{0}-\frac{1}{2}\left(\beta^{2} y^{2}+(-\beta z+h y)^{2}+Z^{2}\right)+\frac{1-\lambda^{2}}{2}\left(h^{2} y^{2}\right. \\
& -2 \beta h y z)-\lambda(-\beta z+h y) Z,
\end{aligned}
$$

now scales independently of resistivity (in particular $|Z|_{\max }$ is independent of $\eta$ ).

Although this solution displays fast energy dissipation characteristics and overcomes the pressure build-up problem it is clearly not a sensible candidate for the energy release mechanism of phenomena such as solar flares. This is because the Ohmic heating now occurs throughout the plasma, rather than in a localized region as observed for solar flares.
Furthermore the dimensionless resistivity of the corona, estimated to be $\eta_{c} \approx 10^{-12}$, implies that the sinusoidal field would have to oscillate over a wavelength of order $10^{3.5} \mathrm{~cm}$ (assuming a global length-scale of the coronal field of order $L_{c} \approx 10^{9.5} \mathrm{~cm}$ ). It is not clear how such rapid spatial oscillations of the field could be initiated or sustained.

\section{CONCLUSIONS}

We have shown that it is possible to construct a number of exact analytic magnetic merging and reconnection solutions in the incompressible limit by making some very simple assumptions about the form of the magnetic field. Our approach has also allowed several new quasi-steady reconnection solutions to be derived. The various solutions are conveniently classified in terms of their energy release scalings with plasma resistivity $\eta$. A key issue, as discussed in Sec. II, is whether any solution has the potential to satisfy the extreme energy release constraints imposed by the solar flare.

More specifically, any flare solution must be able to sustain significant energy release at very small plasma resistivities. This must be done without building up coronal gas pressures that exceed the photospheric magnetoconvection pressures that drive the merging. However, while most of our solutions cannot be regarded as serious flare models, they remain interesting as simple, exact descriptions of the magnetic merging and reconnection processes.

In fact only the planar reconnection solution of Sec. III can be regarded as a potential flare candidate. The Dawson function annihilation model (Sec. III B) is very good at releasing energy - it achieves a super fast rate—but the background pressure required to sustain the merging is prohibitively large $\left(p_{0} \sim \eta^{-1}\right)$ due to the continual increase in field strength near the null as $\eta$ is reduced. This unphysical behavior implies an increasing Ohmic dissipation rate with decreasing resistivity, which can only be avoided by postulating the eventual saturation of the annihilation field. That this saturation must occur follows from the reconnection solutions by the constraint of sub-luminal coronal velocity fields. The key point is that by regarding the Dawson function solution as a component in a fully reconnective model, the pressure problem can be avoided while maintaining energy release rates comparable to a flare. This is true even for classically weak resistivities $\left(\eta_{c}=10^{-12}\right)$. By contrast models which involve the advection of a normal field component by a planar flow (Sec. IV) only release energy very slowly, at the Sweet-Parker rate. The oscillating field solutions of Sec. $\mathrm{V}$ could in principle provide the flare energy, but these involve unphysically rapid spatial oscillations of the field.

In Part II we find that relaxing the constraint of planar flow does not lead to a proliferation of potential flare solutions. Specifically we show that although 3-D solutions can have exotic multiple scalings with resistivity, these do little to ease the severe constraints on an acceptable flare mechanism. 


\section{ACKNOWLEDGMENTS}

The authors wish to thank Alfred Sneyd and Richard Fabling for helpful discussions.

P.G.W. acknowledges the financial support of a New Zealand Foundation for Research in Science and Technology Post-doctoral Fellowship.

${ }^{1}$ D. Biskamp, Phys. Rev. Lett. 237, 181 (1994).

${ }^{2}$ T. G. Forbes and E. R. Priest, Rev. Geophys. 25, 1583 (1987).

${ }^{3}$ F. T. Gratton, M. F. Heyn, H. K. Biernat, R. P. Rijnbeek, and G. Gnavi, J. Geophys. Res. 93, 7318 (1988).

${ }^{4}$ T. D. Phan and B. U. O. Sonnerup, J. Plasma Phys. 44, 525 (1990).
${ }^{5}$ B. P. Besser, H. K. Biernat, and R. P. Rijnbeek, Planet. Space Sci. 38, 411 (1990).

${ }^{6}$ B. P. Besser, H. K. Biernat, and R. P. Rijnbeek, J. Atmos. Terr. Phys. 53, 1081 (1991).

${ }^{7}$ M. Jardine, H. R. Allen, R. E. Grundy, and E. R. Priest, J. Geophys. Res. 97, 4199 (1992).

${ }^{8}$ E. N. Parker, J. Plasma Phys. 9, 49 (1973).

${ }^{9}$ B. U. Ö. Sonnerup and E. R. Priest, J. Plasma Phys. 14, 283 (1975).

${ }^{10}$ I. J. D. Craig and S. M. Henton, Astrophys. J. 450, 280 (1995).

${ }^{11}$ I. J. D. Craig, R. B. Fabling, S. M. Henton, and G. J. Rickard, Astrophys. J. Lett. 455, L197 (1995).

${ }^{12}$ I. J. D. Craig and R. B. Fabling, Astrophys. J. 462, 969 (1996).

${ }^{13}$ P. G. Watson and I. J. D. Craig, Phys. Plasmas 4, 110 (1997).

${ }^{14}$ E. N. Parker, Cosmical Magnetic Fields (Clarendon, Oxford, 1979).

${ }^{15} \mathrm{~J}$. Spanier and K. B. Oldham, An Atlas of Functions (Hemisphere, New York, 1987). 